\title{
BONE AGE -AN IMPORTANT ANATOMICAL TOOL IN THE HANDS OF THE ENDOCRINOLOGIST
}

Ameet Julka, Sandeep Julka, Seema Garg, Bhavana Tiwari, Amarjeet Singh Chhabra,

Sudha Shrivastava
1. Assistant Professor. Department of Anatomy, MGM Medical College, Indore.
2. Consultant Endocrinologist. Synergy Hospital. Indore.
3. Demonstrator. Department of Anatomy, MGM Medical College, Indore.
4. Demonstrator. Department of Anatomy, MGM Medical College, Indore.
5. Assistant Professor. Department of Physiology, MGM Medical College, Indore.
6. Professor \& HOD. Department of Anatomy, MGM Medical College, Indore.

\section{CORRESPONDING AUTHOR}

Dr. Sandeep Julka,

Consultant Endocrinologist,

Synergy Hospital,

Sch No.74-C, Sector-B, Vijay Nagar,

Indore (MP) -452010,

E-mail: sandeep-julka@yahoo.com

Ph: 00919977999687

ABSTRACT: OBJECTIVE: The skeletal maturity of any individual is known as bone age and it can be reliably estimated by a roentgenologic study of osseous development since the appearance and union of the centers of ossification occur in a fairly definite pattern and time sequence from birth to maturity. This is particularly helpful in the clinical workup of children with endocrinological disorders where skeletal and pubertal growth is affected. Therefore the bone age can be advanced, delayed or appropriate for chronological age in various endocrinological disorders. We present cases which affect skeletal maturation in a way that a simple investigation like the bone age estimation can lead to their diagnosis or can help therapeutic interventions. The present study aims to reiterate the importance of bone age in the diagnosis and management of endocrinological disorders. MATERIAL AND METHODS: X rays of both hands of cases and for bone age estimation we used the Greulich - Pyle atlas to accord a bone age to each bone of the hand and obtain an average reading. RESULT: The endocrinological disorders were classified as advanced, delayed and bone age appropriate for chronological age. Cases with advanced bone age were a child with hypothalamic hamartoma and a child with congenital adrenal hyperplasia. Those with delayed bone age were hypothyroidism and growth hormone deficiency. The case with bone age appropriate for chronological age was that of a Turner girl. In each of these cases the bone age helped in the diagnosis and also in the therapeutic decisions. CONCLUSIONS: Assessing bone age through radiographs of both hands graded according to Greulich Pyle method provided the endocrinologists an inexpensive tool to obtain an objective measure of the child's developmental status and also an effective tool for the diagnosis and differential diagnosis of various endocrinological disorders. It also guides the endocrinologist of the timing and effectiveness of the therapeutic measures employed.

KEY WORDS : Bone age, Chronological age, Greulich -Pyle atlas, puberty, precocity

INTRODUCTION: The growing skeleton is sensitive to relatively slight and transient illnesses (endocrine and others). The age of a growing skeleton may fairly reliably be estimated since the 
appearance and union of the centres of ossification occur in a fairly definite pattern and time sequence from birth to maturity. Roentgenologic study of osseous development provides a valuable guide for evaluation of normal and abnormal growth. The skeletal maturity of any individual is known as the Bone Age.

Two criteria are used for determining the bone age of a person by assessing the ossification centres:-

1) The number and size of epiphyseal centres demonstrable at a given chronological age. The time of appearance is specific for each epiphysis of each bone for each sex. So the principles governing their sequence and their site of appearance should be known.

2) The disappearance of the dark line representing the epiphyseal cartilage plate which indicates that the epiphysis has fused to the diaphysis. The sequence of dates of union is remarkably constant and the intervals between them remain proportionately the same in different people1.

In some cases of endocrinopathy, a whole group of ossification centres may fail to appear or show a delayed appearance. Bone age (BA) can be advanced (i.e. bone age reading more than the chronological age), delayed (i.e. bone age reading lesser than the chronological age) or appropriate for chronological age (CA). So Bone age estimation is particularly helpful in the clinical workup of children with growth and pubertal disorders as well as in making treatment related decisions, such as whether to start replacement therapy in a patient with hypogonadism. Bone age is read by endocrinologist or radiologist using radiograph of the hands.

The Greulich - Pyle bone age atlas ${ }^{2}$ consists of representative hand radiograph of boys and girls at all ages with descriptions of indicators of maturity of each bone. The best way to use the system is to accord a bone age reading to each bone in the hand by comparing it with the radiograph that matches best and then obtaining an average reading, rather than by getting an approximate match for the whole radiograph ${ }^{3}$.

Skeletal maturity assessment plays a pivotal role in confirming the diagnosis of normal variants of growth such as familial short stature and constitutional delay of growth, in interpreting hormone tests during puberty and in the diagnosis of precocious puberty or delayed puberty. The present study aims to reiterate the importance of bone age in the differential diagnosis and management of endocrinological disorders.

MATERIAL \& METHODS: X - Rays of both hands of cases and karyotyping incase of chromosomal disorders. We used the Greulich - Pyle atlas ${ }^{2}$ to accord a bone age to each bone of the hand and obtain an average reading.

RESULTS: The endocrinological disorders were classified as 'advanced bone age', 'delayed bone age' and 'bone age appropriate for chronological age'. The following case vignettes demonstrate conditions which affect skeletal maturation in a way that can lead to their diagnosis or can help in their therapeutic interventions.

\section{ADVANCED BONE AGE:}

CASE 1: History - A 2 year old male child presented with history of early development of secondary sexual characters. The child was a product of full term normal vaginal delivery with normal psychomotor milestones. 
Examination -

1. Testicular volume of $8 \mathrm{ml}$ bilaterally

2. Stretched penile length of $6.5 \mathrm{~cm}$ (both high for his age).

Investigations -

$\mathrm{X}$-Rays of both hands for bone age determination.

The bone age of the child as determined by Greulich - Pyle atlas was 7 years as against the chronological age of 2 years. The advanced bone age signifies the rapidly progressive maturation of skeletal system due to systemic exposure to gonadal steroids. GnRH analogue stimulation test confirmed central sexual precocity.

MRI scan of the brain revealed a hypothalamic hamartoma.

Diagnosis - Hypothalamic Hamartoma causing central sexual precocity. (Fig 1, 2a, 2b)

\section{CASE 2}

History - A 4 year 8 month old male child was referred for enlargement of penis. The child did not have any history of episodic prostration or hypotension.

Examination -

1. Testicular volume was $4 \mathrm{ml}$ bilaterally.

2. Stretched penile length was $8 \mathrm{~cm}$ (both advanced for his age).

Investigations -

$\mathrm{X}$ - Ray both hands was done for evaluation of bone age.

The bone age of the child as determined with Greulich - Pyle atlas was 9 years which was advanced for his chronological age of 4 years and 8 months.

Serum 17 hydroxy progesterone was high while serum sodium and potassium were normal.

Diagnosis - Simple Virilizing Congenital Adrenal Hyperplasia (Fig 3a, 3b)

\section{DELAYED BONE AGE}

\section{Case 3}

History- A 15 year old male was referred for evaluation of short stature. The child was a product of full term normal delivery. He also had history of poor scholastic performance.

Investigations -

$\mathrm{X}$ - Rays of both hands were done for evaluation of bone age.

The bone age, determined as mentioned previously was 8 years, against the chronological age of 15 years.

Thyroid Function Tests: TSH was more than $100 \mathrm{IU} / \mathrm{L}$. 
Diagnosis - Primary Hypothyroidism (Fig 4a, 4b)

\section{Case 4}

History - A 13 year old girl was referred for evaluation of short stature.

Examination -

1. The child was short, had frontal bossing, midfacial hypoplasia and crowding of teeth.

Investigations -

X-Rays of both hands were done.

The bone age of the patient was estimated to be 7 years and 10 months against the chronological age of 13 years.

Specialized Tests - Growth hormone stimulation test after priming with oral estrogen demonstrated GH deficiency.

MRI - MRI of the brain showed a pituitary gland of small size.

Diagnosis - Growth Hormone Deficiency (Fig 5, 6a, 6b)

Bone Age Appropriate For Chronological Age

\section{Case 5}

History - A 5 year and 6 months old girl child was referred for short stature. The girl was a product of full term normal vaginal delivery with normal psychomotor milestones.

Examination -

1. There was frontal bossing and increased carrying angle. The girl had proportionate short stature.

Investigations -

$\mathrm{X}$-Rays of both hands demonstrated bone age

equal to the chronological age of 5 years and 6 months.

In view of the normal bone age, a possibility of Turner's Syndrome was kept.

Karyotyping revealed $45 \mathrm{XO}$ chromosomal pattern.

Diagnosis - Turner's Syndrome (Fig 7, 8a, 8b)

DISCUSSION: Bone age is a measure of developmental age, or physiological maturity which represents more truthfully than chronological age how far an individual has progressed towards full maturity. In the clinical setup of hyperandrogenism, the bone age is advanced whereas in hypothyroidism, panhypopituitarism and constitutional delay in puberty, bone age is delayed albeit at different age ranges. Usually, due to high inter observer variation, only a 
difference of 2 years or more is considered relevant when comparing with the chronological age.

Hypothalamic Hamartoma (Fig.1) is a developmental malformation in the region of tuber cinereum and mamillary bodies that typically manifests in the first or second decade of life. Hypothalamic hamartomas may be sessile or pedunculated and are usually attached to the posterior hypothalamus between the tuber cinereum and the mamillary bodies 4 . It is an important cause of precocious puberty in boys. Some children present with gelastic seizures, some with neurosurgical problems and in others it is an incidental finding.

In precocious puberty, the gonadal and related hormones are present abnormally early in quantities sufficient to cause the epiphyses of the various long bones to fuse before growth has continued long enough to permit the attainment of full normal adult stature ${ }^{2}$. The advanced bone age can be retarded by appropriate GnRH analogue therapy which also causes regression of secondary sexual characters. This not only leads to improved adult height in children with precocity but also improved psychological outcomes.

Congenital Adrenal Hyperplasia is due to an enzymatic defect leading to hyperandrogenism and varying degrees of cortisol deficiency. Bone age is severely delayed in hypothyroidism as the thyroid hormones are important pre-requisite for growth and maturation of body. Hypothyroidism in children is the commonest cause of treatable mental retardation. Once the treatment with thyroid hormone replacement is started, there is rapid catch up growth in the child along with advancement of bone age.

In hypogonadal males and females, growth especially growth of the upper and lower extremities continues for an abnormally long period of time and results in characteristically eunuchoid bodily proportions, in which the limbs are abnormally long and the trunk disproportionately short. A markedly slow progression of skeletal development, particularly at puberty is a constant feature of this distressing syndrome.

Bone age estimation in hypogonadal males helps us to time the testosterone replacement therapy. In girls, institution of estrogen replacement therapy accelerates the bone age. It is known that estrogen is responsible for the epiphyseal fusion in both young men and women. Therefore, as in the case of Turner's syndrome, the estrogen replacement therapy is often delayed to allow appropriate growth with growth hormone therapy. Once desired or satisfactory height gain has occurred, therapy with appropriate gonadal steroid is initiated to bring about development of secondary sexual characters. The above mentioned patient of Turner's syndrome has received three years of growth hormone therapy by the endocrinologist and has achieved a resultant growth velocity of almost double the baseline growth velocity.

Bone age helps in the differential diagnosis of the following conditions

- Familial short stature (No delay in Bone Age).

- Constitutional short stature (Mild delay in Bone Age) versus hormonal short stature (Significant delay in Bone Age).

- Hormonal deficiencies (Significant delay in Bone Age) versus syndromic short stature (No delay in Bone Age).

Bone age helps in therapeutic decisions like -

- GnRH analogue therapy for precocity.

- Steroid therapy for Congenital Adrenal Hyperplasia.

- Growth hormone therapy for short stature. 
- Estrogen replacement therapy for Turner's Syndrome

CONCLUSION: Bone age estimation provides an inexpensive tool in the hands of an endocrinologist for diagnosis and management of various endocrinological disorders. In India and other developing countries where majority of patients cannot afford expensive investigations, a simple one like X-Ray hands for bone age can help us narrow down the investigations.

Bone age estimation provides us an objective measure of determining the child's developmental status and to compare this with that of others of the same age and sex and also an additional evidence of the effectiveness of the therapeutic measures employed in the treatment of the child.

\section{REFERENCES:}

1. Halim A. Surface and radiological Anatomy. CBS publishers \& distributors; pp 119

2. Greulich WW, Pyle SI. Radiographic Atlas Of skeletal Development of the hand and wrist. Stanford: Stanford University Press: 1959.

3. Bhatia V, Dabadghao P. Normal growth. In: Desai MP, Menon PSN, Bhatia V (eds). Pediatric Endocrine Disorders. Hyderabad: Orient Longman Private Limited; 2008: 6174.

4. Dennis MS, Melvin MG. Puberty: Ontogeny, neuroendocrinology, physiology and disorders. In:Kronenberg HM, Melmed S, Polonsky KS, Larsen PR (eds). Williams textbook of endocrinology. Philadelphia: Saunders Elsevier; 2011: pp 1054-1201

Fig.1 MRI of the brain showing hypothalamic hamartoma.

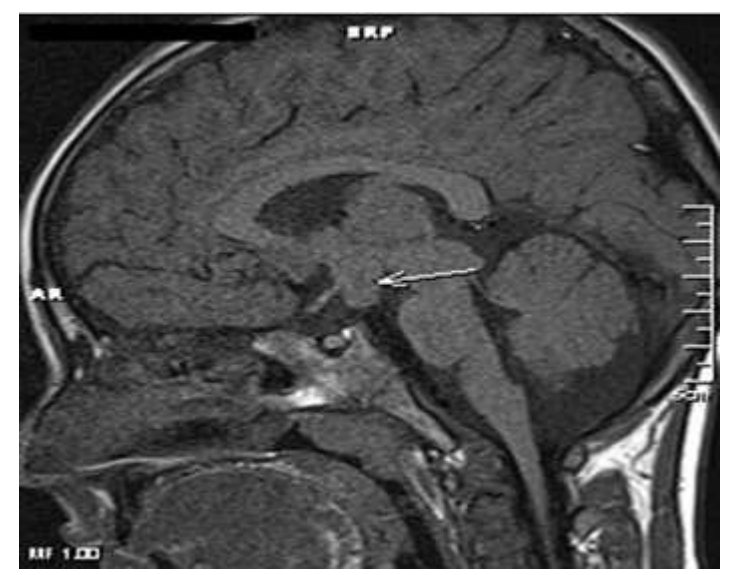


Fig.2a \& Fig.2b X-rays of Control and Case: Advanced bone age - hypothalamic hamartoma

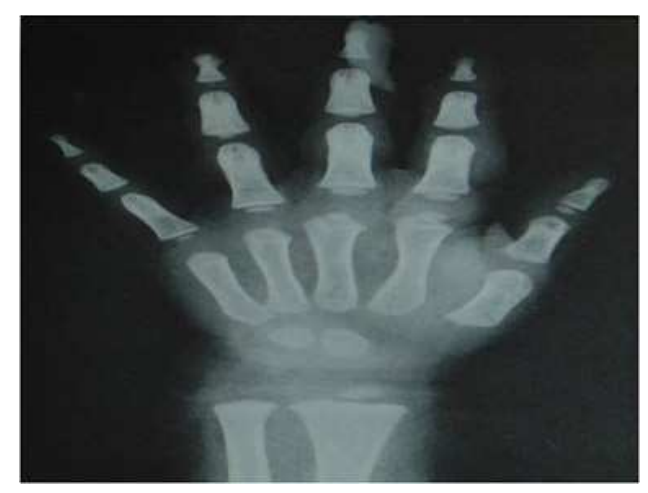

Fig.2a - Chronological Age 2 yrs male (control)

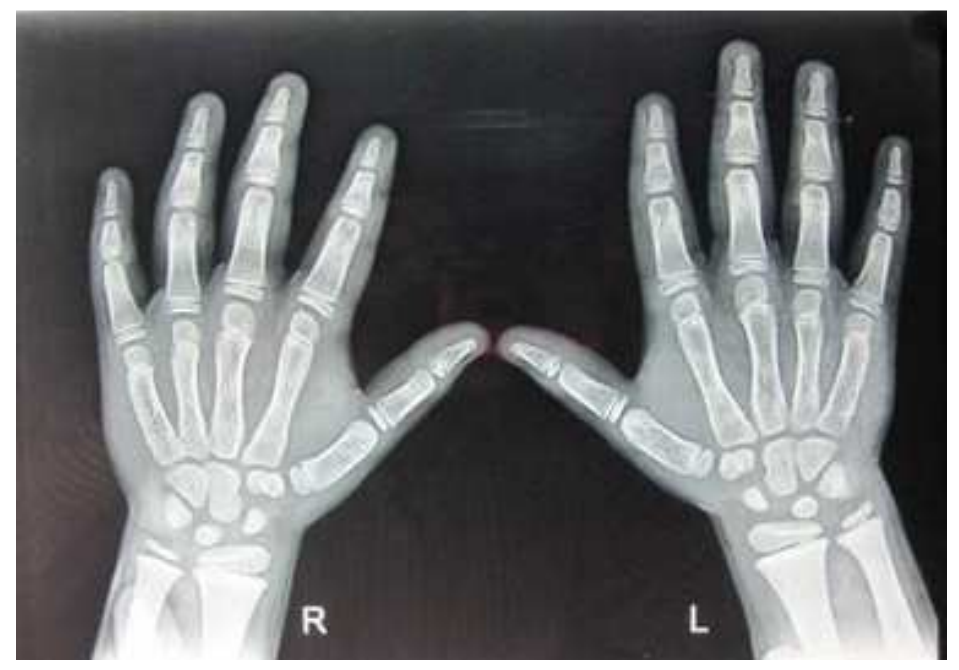

Fig.2b - Bone Age 7 yrs male (case) 


\section{ORIGINAL ARTICLE}

Fig3a \& Fig.3b X-rays of Control and Case: Advanced bone age - congenital adrenal hyperplasia (Case 2).

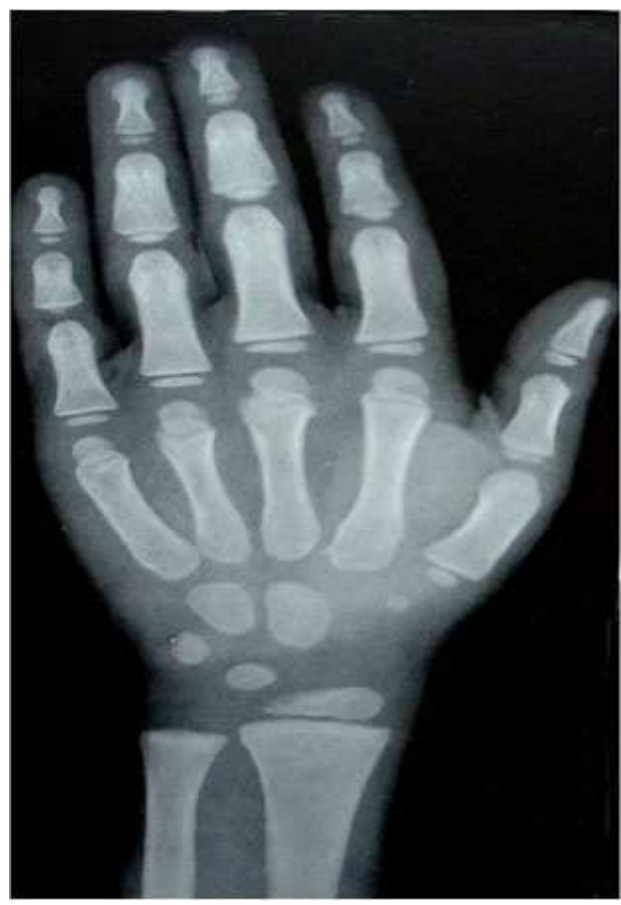

Fig.3a - Chronological Age 4 yr 8 months male (control)

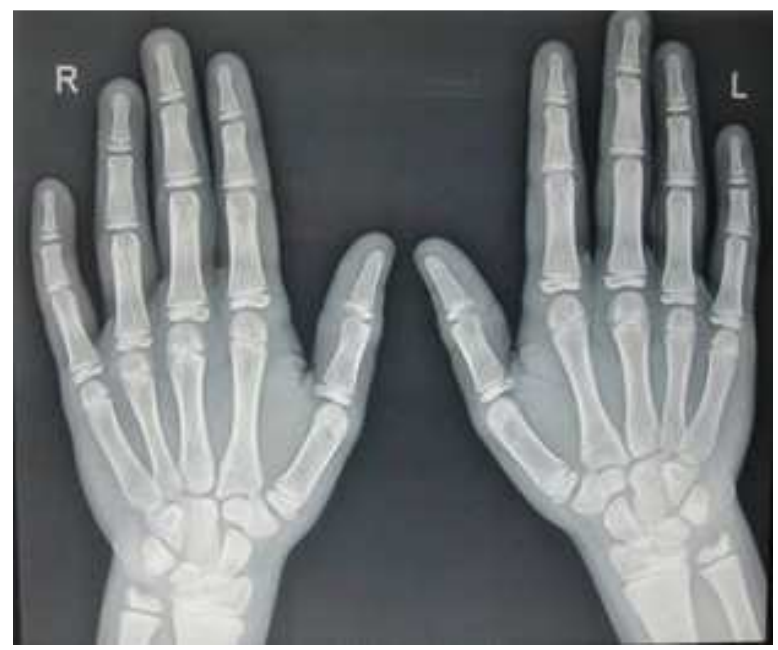

Fig.3b - Bone Age 9 yrs male (case) 


\section{ORIGINAL ARTICLE}

Fig.4a \& Fig.4b X-rays of Control and Case: Delayed bone age - primary hypothyroidism (Case 3).

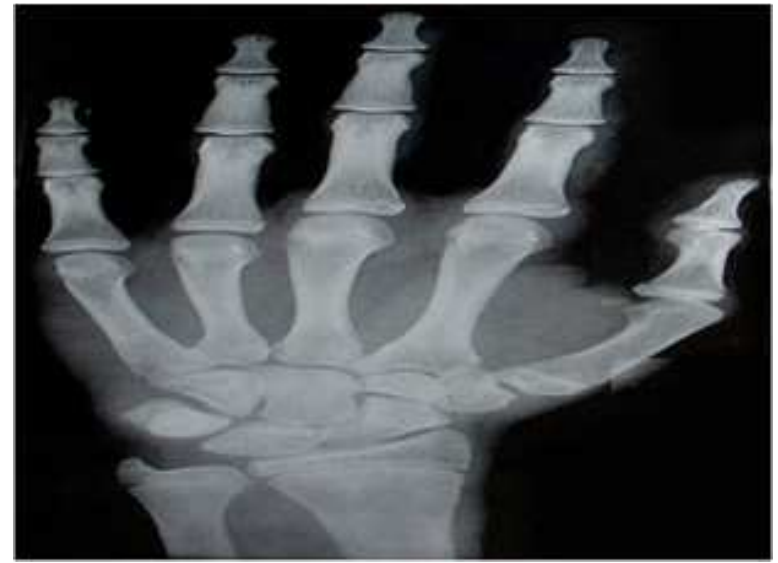

Fig.4a - Chronological Age 15 yrs male (control)

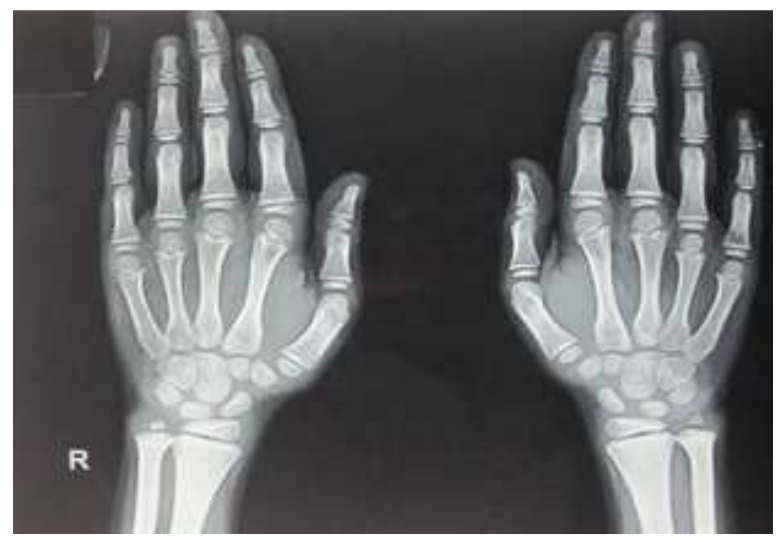

Fig.4a - Bone Age 8 yrs male (case)

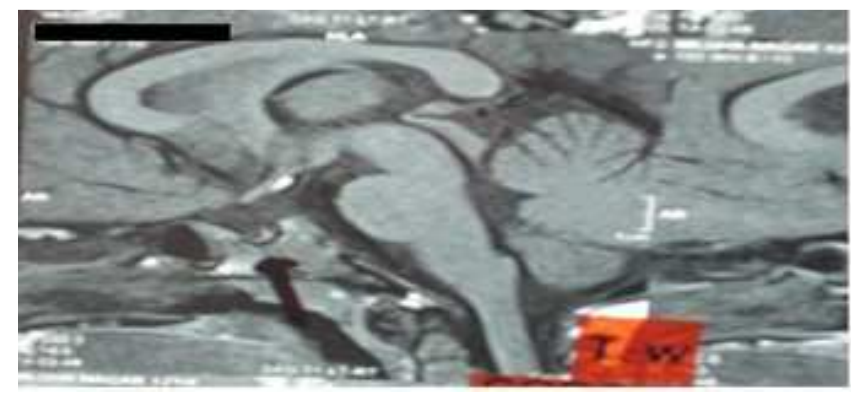

Fig.5 MRI of the brain showing atrophic pituitary gland. 
Fig.6a \& Fig.6b X-rays of Control and Case: Delayed bone age - growth hormone deficiency (Case 4).

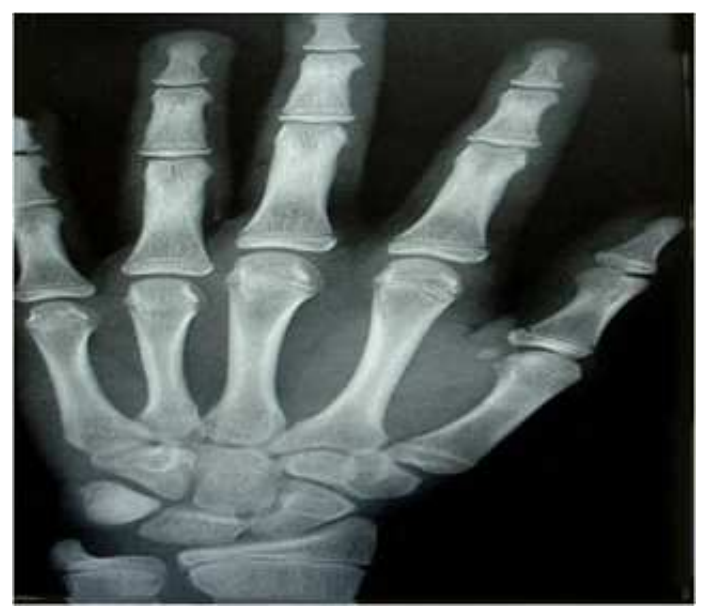

Fig.6a - Chronological Age: 13 yrs- female (Control)

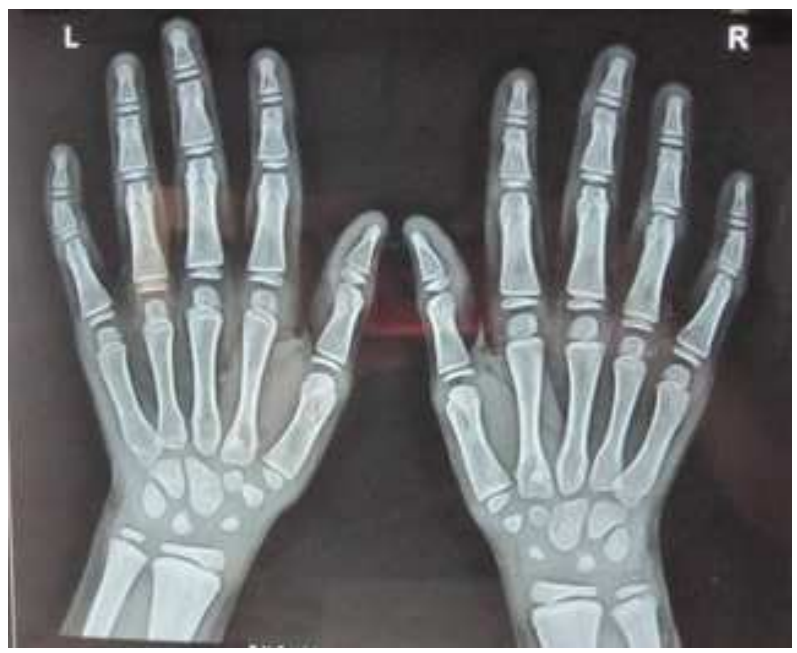

Fig.6b - Bone Age 7 yrs 10 months - female (case) 


\section{ORIGINAL ARTICLE}

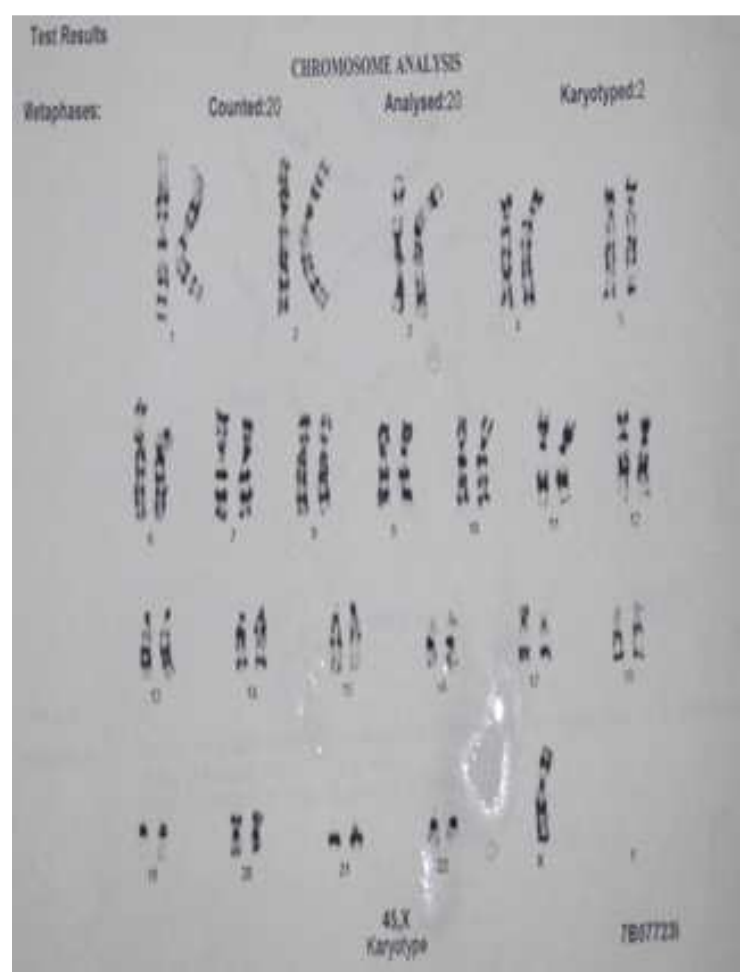

Fig.7 Karyotype revealing 45 XO- Turner's Syndrome

Fig.8a \& Fig.8b X-rays of Control and Case: Bone age appropriate for chronological age Turner's syndrome (Case 5).

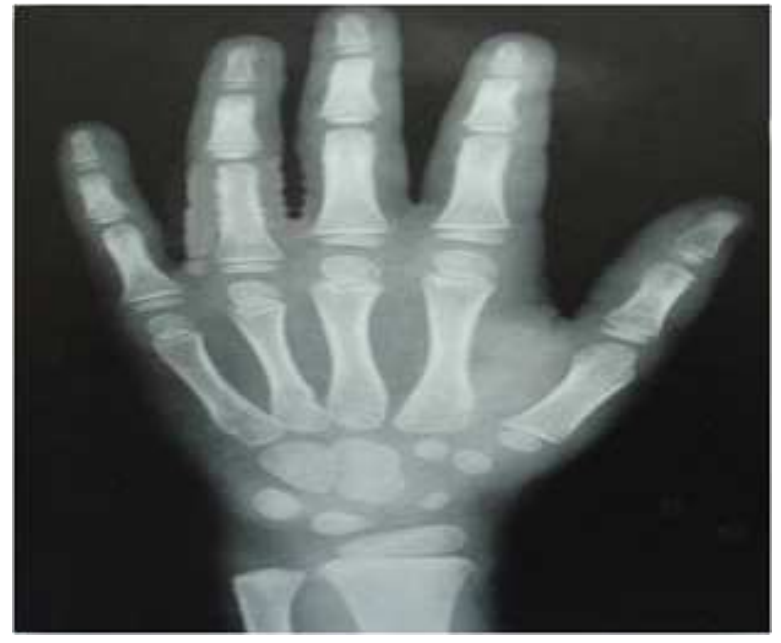

Fig.8a - 5 yrs 6 mths female (Control) 


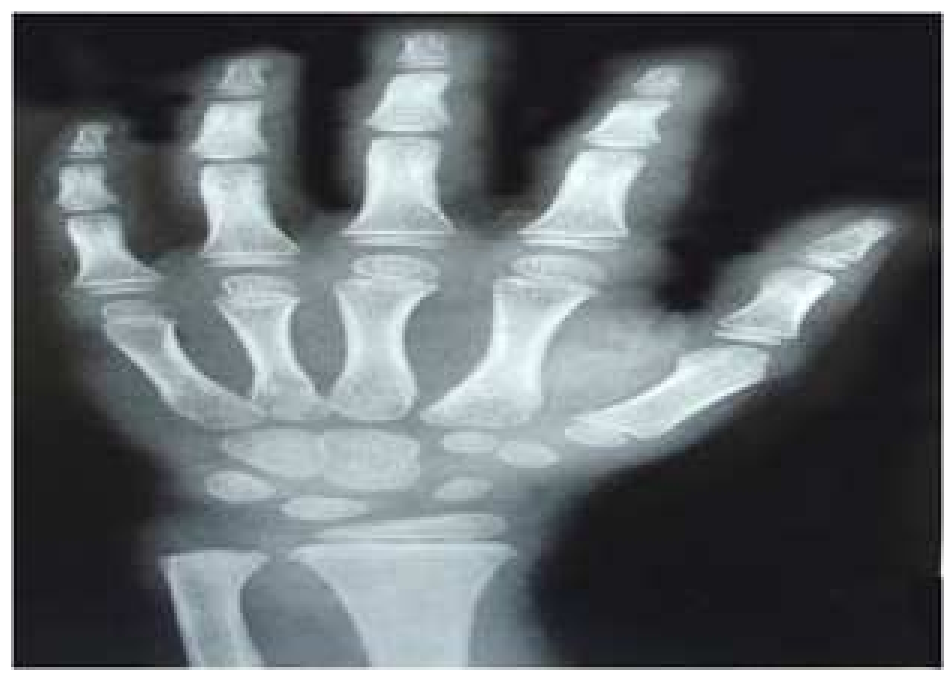

Fig.8b - 5 yrs 6 months female (Case) 